Supporting Information to

\title{
Molecular Dynamics Simulation of Silicon Dioxide Etching by Hydrogen Fluoride Using the Reactive Force Field
}

Dong Hyun Kim, ${ }^{1}$ Seung Jae Kwak, ${ }^{1}$ Jae Hun Jeong, ${ }^{1}$ Suyoung Yoo, ${ }^{2}$ Sang Ki Nam, ${ }^{2}$ YongJoo $\mathrm{Kim}^{3, *}$ and Won Bo Lee ${ }^{1, *}$

${ }^{1}$ School of Chemical and Biological Engineering, Institute of Chemical Processes, Seoul National University, Seoul 08826, Republic of Korea

2 Samsung Electronics, 1-1 Samsungjeonja-ro, Hwaseong, Gyeonggi 18448, Republic of Korea

${ }^{3}$ School of Advanced Materials Engineering, Kookmin University, Seoul 02707, Republic of Korea

E-mail: cjyjee@kookmin.ac.kr (Prof. Y. Kim) and wblee@snu.ac.kr (Prof. W. B. Lee) 

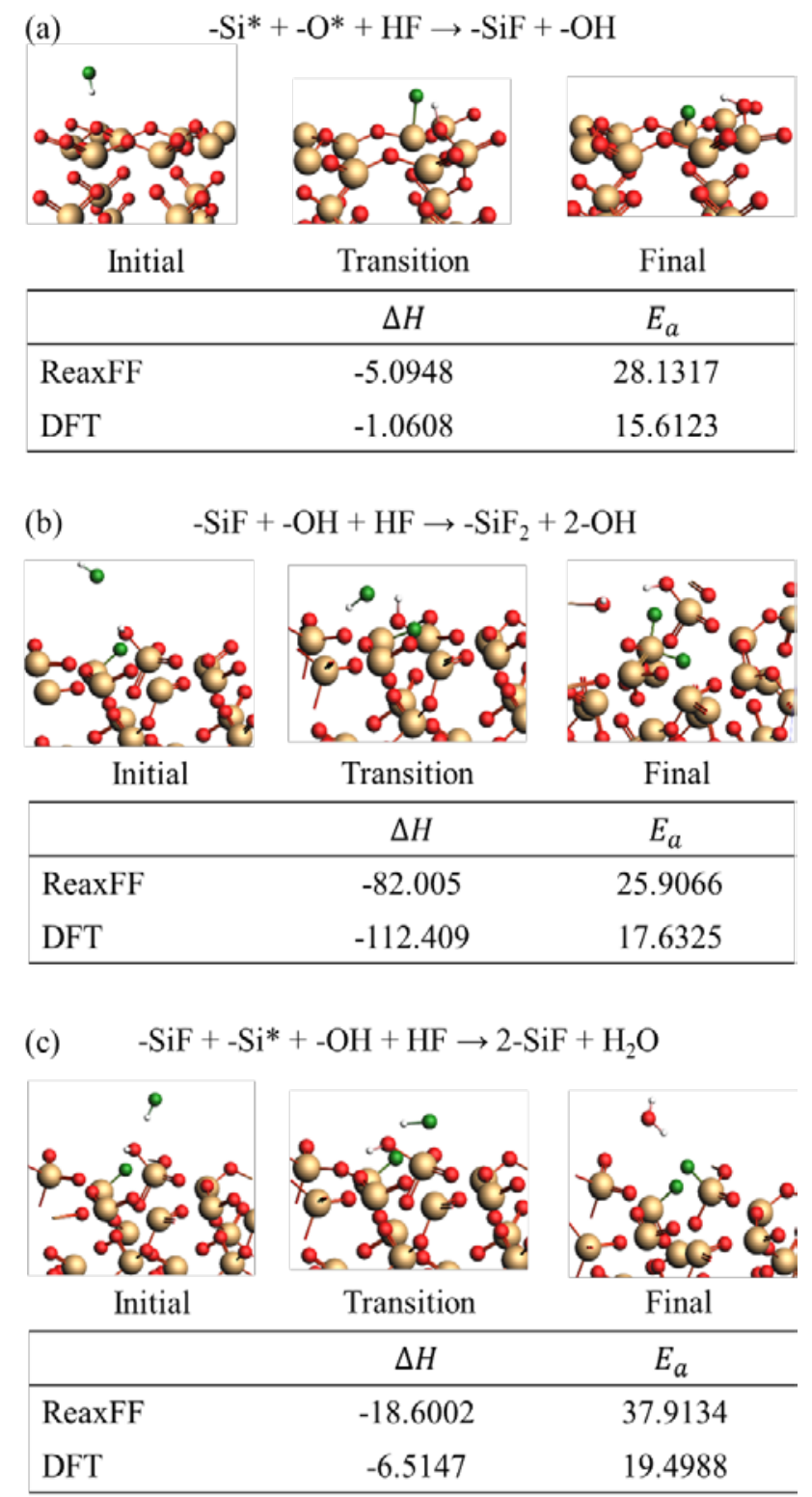

Figure S1. Early stage reactions using $\mathrm{SiO}_{2}$ slab model. (a) HF reacts with surface forming one Si-F bond and one $\mathrm{O}-\mathrm{H}$ bond where $-\mathrm{Si}^{*}$ and $-\mathrm{O} *$ represent reactions sites. (b) HF reacts with surface containing one Si-F bond and one O-H bond forming additional Si-F bond in the same Si atom and additional O-H bond in neighboring Si atom. (c) HF reacts with surface containing one Si-F bond and one O-H bond forming additional Si-F bond in neighboring Si atom and producing $\mathrm{H}_{2} \mathrm{O}$ where $-\mathrm{Si}^{*}$ represents reaction site. 
Table S1. Optimized ReaxFF parameters for Si/O/H/F

50.0000 ! p_boc1 Eq(4c): Overcoordination parameter

9.5469 ! p_boc2 Eq(4d): Overcoordination parameter

26.5405 ! p_coa2 Eq(15): Valency angle conjugation

1.7224 ! p_trip4 Eq(20): Triple bond stabilisation

6.8702 ! p_trip3 Eq(20): Triple bond stabilisation

60.4850 ! k_c2 Eq(19): C2-correction

1.0588 ! p_ovun6 Eq(12): Undercoordination

4.6000 ! p_trip2 Eq(20): Triple bond stabilisation

12.1176 ! p_ovun7 Eq(12): Undercoordination

13.3056 ! p_ovun8 Eq(12): Undercoordination

-70.5044 ! p_trip1 Eq(20): Triple bond stabilization

0.0000 ! Lower Taper-radius (must be 0)

10.0000 ! R_cut Eq(21): Upper Taper-radius

2.8793 ! p_fe1 Eq(6a): Fe dimer correction

33.8667 ! p_val6 Eq(13c): Valency undercoordination 6.0891 ! p_lp1 Eq(8): Lone pair param

1.0563 ! p_val9 Eq(13f): Valency angle exponent

2.0384 ! p_val10 Eq(13g): Valency angle parameter

6.1431 ! p_fe2 Eq(6a): Fe dimer correction

6.9290 ! p_pen2 Eq(14a): Double bond/angle param 
0.3989 ! p_pen3 Eq(14a): Double bond/angle param

3.9954 ! p_pen4 Eq(14a): Double bond/angle param

-2.4837 ! p_fe3 Eq(6a): Fe dimer correction

5.7796 ! p_tor2 Eq(16b): Torsion/BO parameter

10.0000 ! p_tor3 Eq(16c): Torsion overcoordination

1.9487! p_tor4 Eq(16c): Torsion overcoordination

-1.2327 ! p_elho Eq(26a): electron-hole interaction

2.1645 ! p_cot2 Eq(17b): Conjugation if tors13=0

1.5591 ! p_vdW1 Eq(23b): vdWaals shielding

0.1000 ! Cutoff for bond order $(* 100)$

2.1365 ! p_coa4 Eq(15): Valency angle conjugation

0.6991 ! p_ovun4 Eq(11b): Over/Undercoordination

50.0000 ! p_ovun3 Eq(11b): Over/Undercoordination

1.8512 ! p_val8 Eq(13d): Valency/lone pair param

0.5000 ! X_soft Eq(25): ACKS2 softness for X_ij

20.0000 ! d Eq(23d): Scale factor in lg-dispersion

5.0000 ! p_val Eq(27): Gauss exponent for electrons

0.0000 ! $1 \mathrm{Eq}(13 \mathrm{e})$ : disable undecoord in val angle

2.6962 ! p_coa3 Eq(15): Valency angle conjugation

$6 \quad$ ! Number of atoms;

R(sigma);Valence;Atomic;R(VdW);E(VdW);EEM;R(pi);Num;Alpha(VdW);Gamma; ! valency;p(ovun5);p_xel2;EEM;EEM;Type;R(pi2);E(lp);HeatForm;p_boc4; ! p_boc3;p_boc5;Softcut(ACKS2);Alpha(e-reax);p_ovun2;p_val3;Beta(e- 
reax);Val'^boc;p_val5;R(core);

\begin{tabular}{|c|c|c|c|c|c|c|c|c|}
\hline \multirow[t]{4}{*}{$\mathrm{H}$} & 0.8707 & 1.0000 & 1.0080 & 1.4947 & 0.0785 & 0.7599 & -0.1000 & 1.0000 \\
\hline & 8.1936 & 33.9645 & 1.0000 & \multicolumn{2}{|c|}{0.0000121 .1250} & 3.6146 & 9.8692 & 1.0000 \\
\hline & -0.1000 & 0.0000 & 59.3302 & 3.3628 & 2.2226 & 0.0022 & 1.0698 & 0.0000 \\
\hline & -19.4527 & 4.4710 & 1.0338 & 1.0000 & 2.8793 & 0.0000 & 0.0000 & 0.0000 \\
\hline \multirow[t]{4}{*}{$\mathrm{O}$} & 1.4075 & 2.0000 & 15.9990 & 1.4115 & 0.0977 & 1.0950 & 1.0878 & 6.0000 \\
\hline & 9.6471 & 16.6038 & 4.0000 & \multicolumn{2}{|c|}{38.3368116 .0768} & 7.9705 & 8.8485 & 2.0000 \\
\hline & 0.9050 & 27.8683 & -1.0116 & 2.3988 & 4.0090 & 0.0028 & 0.9745 & 0.0000 \\
\hline & -3.3587 & 3.2099 & 1.0493 & 4.0000 & 2.9225 & 0.0000 & 0.0000 & 0.0000 \\
\hline \multirow[t]{4}{*}{$\mathrm{N}$} & 1.2335 & 3.0000 & 14.0000 & 2.2402 & 0.1057 & 0.5903 & 1.3517 & 5.0000 \\
\hline & 9.6729 & 12.7207 & 4.0000 & \multicolumn{2}{|c|}{31.5285100 .0000} & 6.1159 & 7.4005 & 2.0000 \\
\hline & 1.0345 & 0.08681 & 129.5034 & 0.6274 & 7.0842 & 2.7565 & 0.9745 & 0.0000 \\
\hline & -2.0090 & 4.2982 & 1.0183 & 4.0000 & 2.8793 & 0.0000 & 0.0000 & 0.0000 \\
\hline \multirow[t]{4}{*}{$\mathrm{Si}$} & 1.9733 & 4.0000 & 28.0600 & 2.0654 & 0.1928 & 0.7500 & 1.2223 & 4.0000 \\
\hline & 12.1892 & 1.2539 & 4.0000 & \multicolumn{2}{|c|}{21.4869139 .9309} & 1.8038 & 6.0009 & 0.0000 \\
\hline & -1.0000 & \multicolumn{2}{|c|}{0.0000104 .0224} & 8.2570 & 5.2300 & 0.8381 & 0.8563 & 0.0000 \\
\hline & -4.8039 & 2.0754 & 1.0338 & 4.0000 & 2.7786 & 0.0000 & 0.0000 & 0.0000 \\
\hline \multirow[t]{4}{*}{$\mathrm{F}$} & 1.1978 & 1.0000 & 18.9984 & 1.5723 & 0.1254 & 0.3000 & -0.1000 & 7.0000 \\
\hline & 11.3841 & 9.4986 & 4.0000 & 7.3951 & 0.2000 & 9.0000 & 15.0000 & 0.0000 \\
\hline & -1.0000 & 34.9703 & 17.9169 & 7.9796 & 5.1771 & 1.0561 & 0.0000 & 0.0000 \\
\hline & -5.3030 & 3.1624 & 1.0493 & 4.0000 & 2.4240 & 0.0000 & 0.0000 & 0.0000 \\
\hline $\mathrm{X}$ & -0.1000 & 2.0000 & 1.0080 & 2.0000 & 0.0000 & 1.0000 & -0.1000 & 6.0000 \\
\hline
\end{tabular}




\section{S6}

$$
\begin{array}{rrrrrrrr}
10.0000 & 2.5000 & 4.0000 & 0.0000 & 0.0000 & 8.5000 & 1.5000 & 0.0000 \\
-0.1000 & 0.0000 & 127.6226 & 8.7410 & 13.3640 & 0.6690 & 0.9745 & 0.0000 \\
-11.0000 & 2.7466 & 1.0338 & 6.2998 & 2.8793 & 0.0000 & 0.0000 & 0.0000
\end{array}
$$

15 ! Number of bonds; E(Sigma);E(Pi);E(Pi2);p_be1;p_bo5;13corr;p_bo6;p_ovun1;

! p_be2;p_bo3;p_bo4;Reserved;p_bo1;p_bo2;ovcoord;p_xel1;

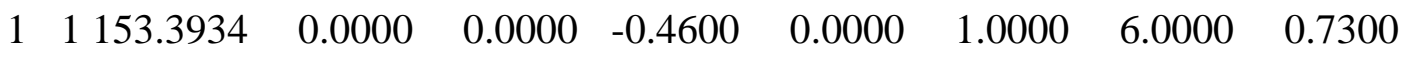

$\begin{array}{llllllll}6.2500 & 1.0000 & 0.0000 & 1.0000 & -0.0790 & 6.0552 & 0.0000 & 0.0000\end{array}$

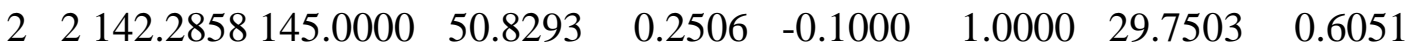

$\begin{array}{llllllll}0.3451 & -0.1055 & 9.0000 & 1.0000 & -0.1225 & 5.5000 & 1.0000 & 0.0000\end{array}$

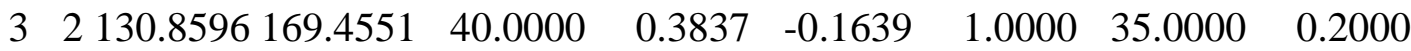

$\begin{array}{llllllll}1.0000 & -0.3579 & 7.0004 & 1.0000 & -0.1193 & 6.8773 & 1.0000 & 0.0000\end{array}$

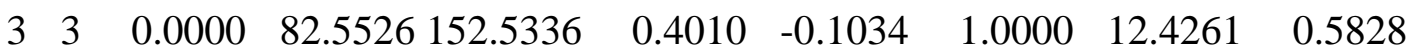

$\begin{array}{llllllll}0.1578 & -0.1509 & 11.9186 & 1.0000 & -0.0861 & 5.4271 & 1.0000 & 0.0000\end{array}$

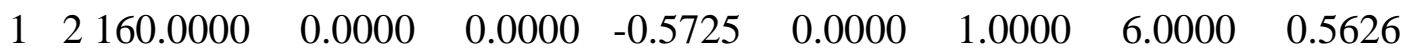

$\begin{array}{llllllll}1.1150 & 1.0000 & 0.0000 & 0.0000 & -0.0920 & 4.2790 & 0.0000 & 0.0000\end{array}$

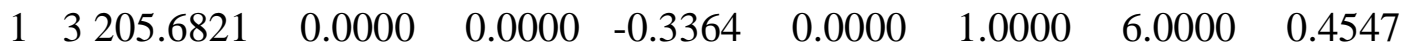

$\begin{array}{llllllll}6.9381 & 1.0000 & 0.0000 & 1.0000 & -0.1310 & 5.5195 & 0.0000 & 0.0000\end{array}$

$\begin{array}{lllllllll}1 & 4250.0000 & 0.0000 & 0.0000 & -0.7128 & 0.0000 & 1.0000 & 6.0000 & 0.1186\end{array}$

$\begin{array}{llllllll}18.5790 & 1.0000 & 0.0000 & 1.0000 & -0.0731 & 7.4983 & 0.0000 & 0.0000\end{array}$

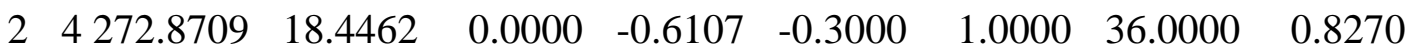

$\begin{array}{llllllll}10.2334 & -0.5495 & 29.9954 & 1.0000 & -0.1277 & 7.5863 & 1.0000 & 0.0000\end{array}$

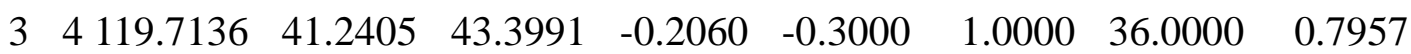




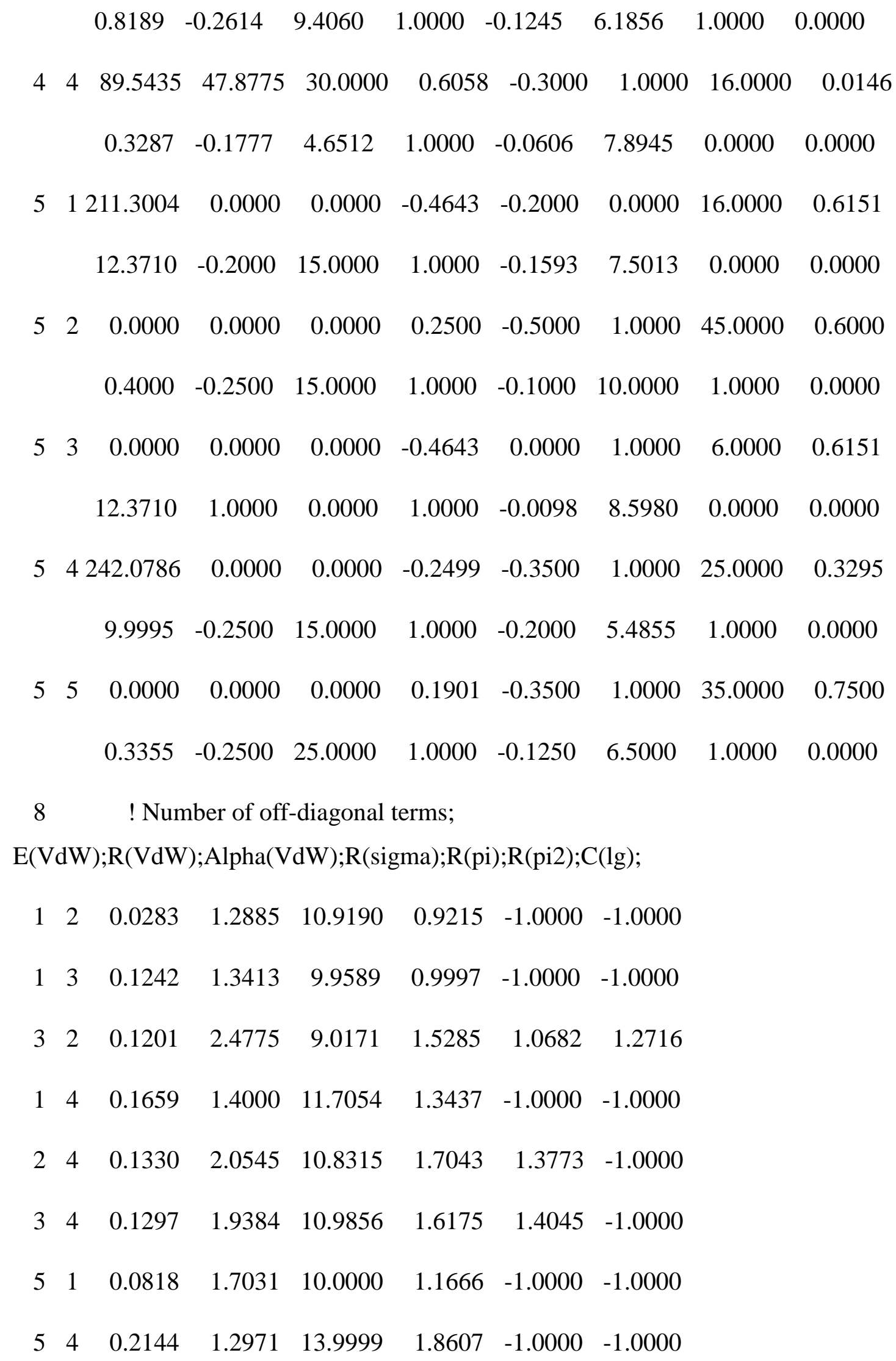


47 ! Number of angles; Theta0;p_val1;p_val2;p_coa1;p_val7;p_pen1;p_val4;

$\begin{array}{llllllllll}1 & 1 & 1 & 0.0000 & 27.9213 & 5.8635 & 0.0000 & 0.0000 & 0.0000 & 1.0400\end{array}$

$\begin{array}{llllllllll}2 & 2 & 2 & 89.9934 & 17.9465 & 1.7798 & 0.0000 & 2.9881 & 0.0000 & 1.0538\end{array}$

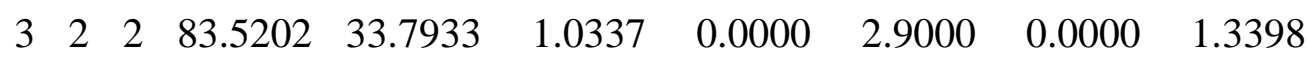

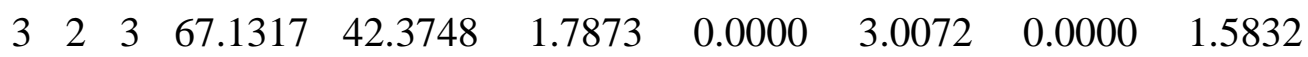

$\begin{array}{llllllllll}1 & 2 & 2 & 75.6935 & 50.0000 & 2.0000 & 0.0000 & 1.0000 & 0.0000 & 1.1680\end{array}$

$\begin{array}{llllllllll}1 & 2 & 3 & 72.7348 & 20.1071 & 7.5000 & 0.0000 & 0.1000 & 0.0000 & 1.0746\end{array}$

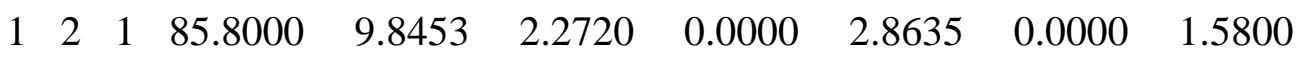

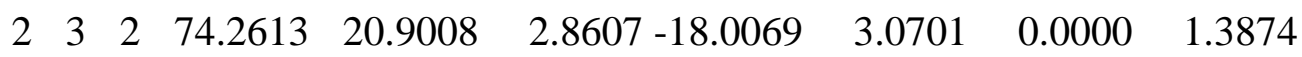

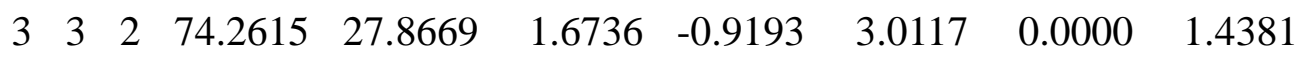

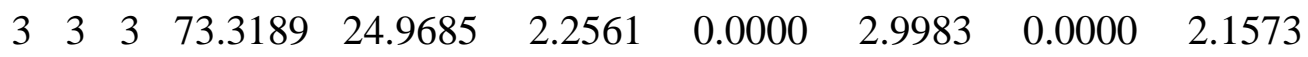

$\begin{array}{llllllllll}1 & 3 & 2 & 74.5739 & 45.0000 & 1.4078 & 0.0000 & 0.3956 & 0.0000 & 3.0000\end{array}$

$\begin{array}{llllllllll}1 & 3 & 3 & 79.7136 & 45.0000 & 0.5316 & 0.0000 & 0.5437 & 0.0000 & 1.0000\end{array}$

$\begin{array}{llllllllll}1 & 3 & 1 & 78.6680 & 6.9060 & 3.7869 & 0.0000 & 0.0223 & 0.0000 & 2.0220\end{array}$

$\begin{array}{llllllllll}2 & 1 & 2 & 0.0000 & 15.0000 & 2.8900 & 0.0000 & 0.0000 & 0.0000 & 2.8774\end{array}$

$\begin{array}{llllllllll}3 & 1 & 2 & 0.0000 & 1.0574 & 0.1000 & 0.0000 & 0.0000 & 0.0000 & 2.7676\end{array}$

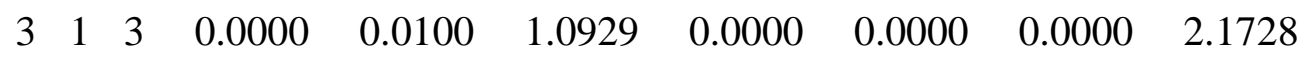

$\begin{array}{llllllllll}1 & 1 & 2 & 0.0000 & 8.5744 & 3.0000 & 0.0000 & 0.0000 & 0.0000 & 1.0421\end{array}$

$\begin{array}{llllllllll}1 & 1 & 3 & 0.0000 & 0.0019 & 6.0000 & 0.0000 & 0.0000 & 0.0000 & 1.0400\end{array}$

$\begin{array}{llllllllll}4 & 4 & 4 & 77.4746 & 39.1276 & 0.8607 & 0.0000 & 0.0024 & 0.0000 & 1.2899\end{array}$

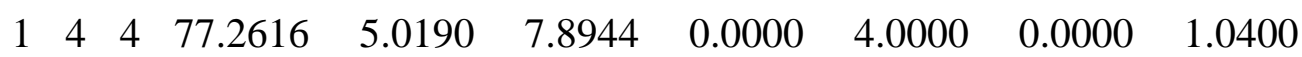

$\begin{array}{llllllllll}1 & 4 & 1 & 75.7983 & 14.4132 & 2.8640 & 0.0000 & 4.0000 & 0.0000 & 1.0400\end{array}$

$\begin{array}{llllllllll}2 & 4 & 4 & 99.8997 & 26.6610 & 2.1237 & 0.0000 & 0.0100 & 0.0000 & 1.4341\end{array}$ 


\begin{tabular}{|c|c|c|c|c|c|c|c|c|c|}
\hline & $4:$ & 2 & 73.6998 & 40.0000 & 1.8782 & 0.0000 & 4.0000 & 0.0000 & 1.1290 \\
\hline & 4 & 2 & 98.2184 & 38.9429 & 0.7727 & 0.0000 & 1.1658 & 0.0000 & 2.2641 \\
\hline & 2 & 4 & 39.2858 & 1.3068 & 5.6478 & 0.0000 & 3.8972 & 0.0000 & 3.0000 \\
\hline & 2 & 4 & 79.2126 & 4.8973 & 8.0000 & 0.0000 & 1.0859 & 0.0000 & 2.1209 \\
\hline & 2 & 4 & 82.7397 & 32.1198 & 1.8862 & 0.0000 & 0.1058 & 0.0000 & 1.5443 \\
\hline & 1 & 4 & 0.0000 & 47.1300 & 6.0000 & 0.0000 & 1.6371 & 0.0000 & 1.0400 \\
\hline & 1 & 4 & 0.0000 & 27.4206 & 6.0000 & 0.0000 & 1.6371 & 0.0000 & 1.0400 \\
\hline & 1 & 4 & 0.0000 & 7.0550 & 3.9236 & 0.0000 & 1.6371 & 0.0000 & 1.0400 \\
\hline 3 & 4 & 4 & 60.6199 & 17.7559 & 1.0576 & 0.0000 & 2.1459 & 0.0000 & 1.0400 \\
\hline & 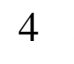 & 3 & 74.1294 & 20.6494 & 2.1244 & 0.0000 & 0.7689 & 0.0000 & 1.0400 \\
\hline 3 & 4 & 2 & 57.0650 & 9.4985 & 0.3423 & 0.0000 & 0.7689 & 0.0000 & 1.0400 \\
\hline & 3 & & 24.1137 & 1.7457 & 0.2198 & 0.0000 & 4.1125 & 0.0000 & 1.0400 \\
\hline 1 & 4 & 3 & 68.7410 & 15.5851 & 1.8545 & 0.0000 & 0.8613 & 0.0000 & 1.0400 \\
\hline & 3 & & 80.9040 & 4.0560 & 1.2284 & 0.0000 & 1.6982 & 0.0000 & 1.0400 \\
\hline 3 & 3 & 4 & 60.0000 & 10.0000 & 0.7500 & 0.0000 & 1.0000 & 0.0000 & 1.0400 \\
\hline & 3 & & 69.8728 & 32.7155 & 1.5875 & 0.0000 & 2.2466 & 0.0000 & 1.0400 \\
\hline 3 & 2 & 4 & 69.8728 & 27.1273 & 1.5875 & 0.0000 & 2.2466 & 0.0000 & 1.0400 \\
\hline & 1 & 4 & 0.0000 & 31.0427 & 4.5625 & 0.0000 & 1.6371 & 0.0000 & 1.0400 \\
\hline 5 & 4 & 4 & 79.7014 & 16.9791 & 1.6839 & 0.0000 & 1.0944 & 0.0000 & 1.0500 \\
\hline & 4 & & 90.9986 & 68.2397 & 4.5675 & 0.0000 & 4.0451 & 0.0000 & 1.0000 \\
\hline 4 & 5 & 4 & 0.0000 & 42.3353 & 7.1082 & 0.0000 & 1.3635 & 0.0000 & 1.0840 \\
\hline b & 5 & 4 & 0.0000 & 10.0000 & 2.0000 & 0.0000 & 1.0000 & 0.0000 & 1.2500 \\
\hline & 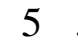 & & 0.0000 & 10.0000 & 2.0000 & 0.0000 & 1.0000 & 0.0000 & 1.2500 \\
\hline
\end{tabular}




$$
\begin{array}{llllllllll}
5 & 1 & 5 & 0.0000 & 0.0000 & 0.0000 & 0.0000 & 0.0000 & 0.0000 & 1.0000 \\
5 & 4 & 2 & 28.9834 & 39.9921 & 1.2589 & 0.0000 & 2.1212 & 0.0000 & 1.0000
\end{array}
$$

14 ! Number of torsions; Theta0;p_val1;p_val2;p_coa1;p_val7;p_pen1;p_val4;

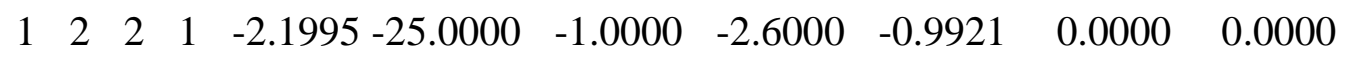

$\begin{array}{lllllllllll}1 & 2 & 2 & 2 & -2.5000 & 43.1840 & -0.6826 & -6.6539 & -1.2407 & 0.0000 & 0.0000\end{array}$

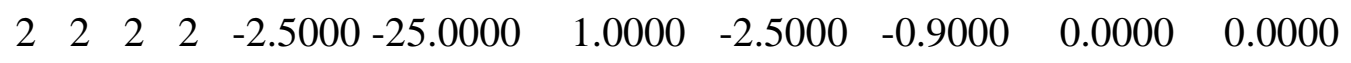

$\begin{array}{lllllllllll}0 & 1 & 1 & 0 & 0.0000 & 0.0000 & 0.0000 & 0.0000 & 0.0000 & 0.0000 & 0.0000\end{array}$

$\begin{array}{lllllllllll}0 & 1 & 2 & 0 & 0.0000 & 0.1000 & 0.0200 & -2.5415 & 0.0000 & 0.0000 & 0.0000\end{array}$

$\begin{array}{lllllllllll}0 & 2 & 2 & 0 & 0.5511 & 25.4150 & 1.1330 & -5.1903 & -1.0000 & 0.0000 & 0.0000\end{array}$

$\begin{array}{lllllllllll}0 & 1 & 3 & 0 & 0.0000 & 0.1032 & 0.3000 & -5.0965 & 0.0000 & 0.0000 & 0.0000\end{array}$

$\begin{array}{lllllllllll}0 & 3 & 2 & 0 & 1.1397 & 61.3225 & 0.5139 & -3.8507 & -2.7831 & 0.0000 & 0.0000\end{array}$

$\begin{array}{lllllllllll}0 & 3 & 3 & 0 & 0.7265 & 44.3155 & 1.0000 & -4.4046 & -2.0000 & 0.0000 & 0.0000\end{array}$

$\begin{array}{lllllllllll}1 & 4 & 4 & 1 & 0.0000 & 0.0000 & 0.0640 & -2.4426 & 0.0000 & 0.0000 & 0.0000\end{array}$

$\begin{array}{lllllllllll}1 & 4 & 4 & 4 & 0.0000 & 0.0000 & 0.1587 & -2.4426 & 0.0000 & 0.0000 & 0.0000\end{array}$

$\begin{array}{lllllllllll}0 & 1 & 4 & 0 & 0.0000 & 0.0000 & 0.1200 & -2.4847 & 0.0000 & 0.0000 & 0.0000\end{array}$

$\begin{array}{lllllllllll}0 & 3 & 4 & 0 & 0.0000 & 0.0000 & 0.0000 & -2.4426 & 0.0000 & 0.0000 & 0.0000\end{array}$

$\begin{array}{lllllllllll}0 & 5 & 5 & 0 & 4.0000 & 45.8264 & 0.9000 & -4.0000 & 0.0000 & 0.0000 & 0.0000\end{array}$

$5 \quad$ ! Number of hydrogen bonds;

Theta0;p_val1;p_val2;p_coa1;p_val7;p_pen1;p_val4;

$\begin{array}{lllllll}2 & 1 & 2 & 2.1200 & -3.5800 & 1.4500 & 19.5000\end{array}$

$\begin{array}{lllllll}2 & 1 & 3 & 2.1215 & -7.5000 & 1.4500 & 19.5000\end{array}$

$\begin{array}{lllllll}3 & 1 & 2 & 1.7500 & -4.3286 & 1.4500 & 19.5000\end{array}$

$\begin{array}{lllllll}3 & 1 & 3 & 2.4000 & -2.3575 & 1.4500 & 19.5000\end{array}$ 
$\begin{array}{lllllll}5 & 1 & 2 & 2.1200 & -2.0000 & 1.4500 & 19.5000\end{array}$ 